\title{
A Rare Case of Pneumonia and Haemoptysis Caused by Citrobactor Freundii in a Young Immunocompetent Male
}

\author{
Sushil Upadhyay \\ Senior Consultant Chest Physician, Holy Family Hospital, Okhla Road, Delhi
}

\begin{abstract}
Citrobacter infections are often nosocomially acquired, seen in patients with significant underlying diseases and isolates are commonly drug-resistant.A rare clinical syndrome of haemoptysis with underlying pneumonia caused by Citrobactor freundii in a immunocompetent young male is presented.
\end{abstract}

Key Words: Citrobactor, Pneumonia, Immunocompetent

\section{Introduction}

Citrobacter genus belongs to family of Enterobacteriacea and comprises of 11 different species of straight, facultative anaerobic, motile,Gram-negative bacilli which are oxidase negative and utilize citrate as sole carbon source ${ }^{1}$. They are commonly found in water, soil, food, and the intestinal tracts of animals and humans ${ }^{2}$. Citrobacter infections typically occur in hospital settings in patients with multiple comorbidities and seldom cause disease in the general population ${ }^{3}$.Urinary tract is the most common site of infection and pneumonia is reported as least common form of infection by Citrobactor species $^{4}$. Community acquired human infections by Citrobactor species are extremely rare and occur at the extremes of age or in the immunocompromised ${ }^{5}$ It has been rarely cultured in the bronchoscopy specimen done for the evaluation of hemoptysis ${ }^{6}$.The case is being reported because of extremely rare combination of immunocompetent host from community setting presenting with haemoptysis,diagnosed as right upper lobe pneumonia on imaging,further confirmed by bronchoscopy and finally yielding Citrobactor freundi on culture of bronchial washings.

\section{Case Report}

Forty four year old male presented in emergency department(ED) with hemoptysis.He complained of one episode of fever 5 days prior followed by expectoration of blood from mouth.Quantity of blood varied between 1 tea-spoon full to 1 table spoon per event which occurred 2-3 times in a day.He had no past medical or surgical
history.He was admitted under general physician. Investigations revealed normal hemogram,renal and liver biochemistries, coagulation parameters.Chest xray(fig1) showed right upper zone hazy airspace opacity.CT thorax(Fig2) confirmed small patch of consolidation in posterior segment of right upper lobe .He received amoxiclav and azithromycin with haemostatics and pantoprazole.He was referred to respiratory specialist for bronchoscopy.Bronchoscopy(Fig3) showed scanty bleeding from right upper lobe posterior segment without any sign of inflammation.Bronchial washings were sent for detailed investigations.Patient was discharged the very next day on oral amoxyclav as he was asymptomatic. However he presented again with haemoptysis 2 days after leaving the hospital.Chest xray revealed same RUZ opacity.Respiratory review was solicited. Bronchial washing which were sent on previous admission were now available.ZN stain and Gene Xpert were negative for Mycobacterium tuberculosis.Cytology was negative for malignant cells.Bacterial culture had grown Citrobactor freundii which was resistant to betalacatam/ lacatamase,cefalosporins,aminogycosides and even carbapenems.It was sensitive only to quinolones, tetracycline, colistin, tigicycline and cotrimoxazole (Fig5).Antibiotics were revised and levofloxacin was given.He was discharged on oral tab of $750 \mathrm{mg}$ of Levofloxacin once a day.Patient was followed up in out patient department after 5 days.He was asymptomatic and repeat chest xray(Fig4) showed resolved RUZ opacity. 

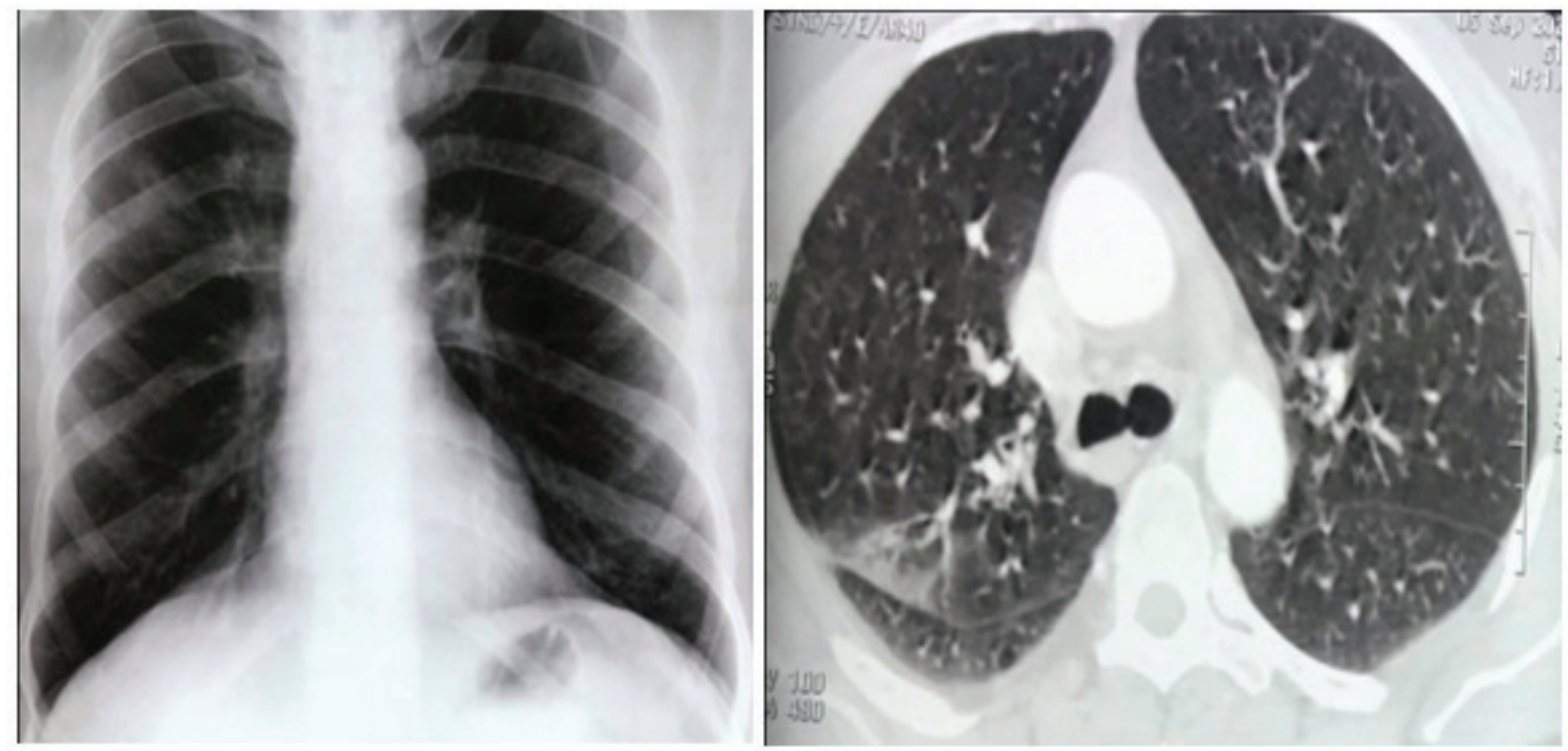

Fig( 1\&2) Chest Xray and CT scan:RUL faint consolidation

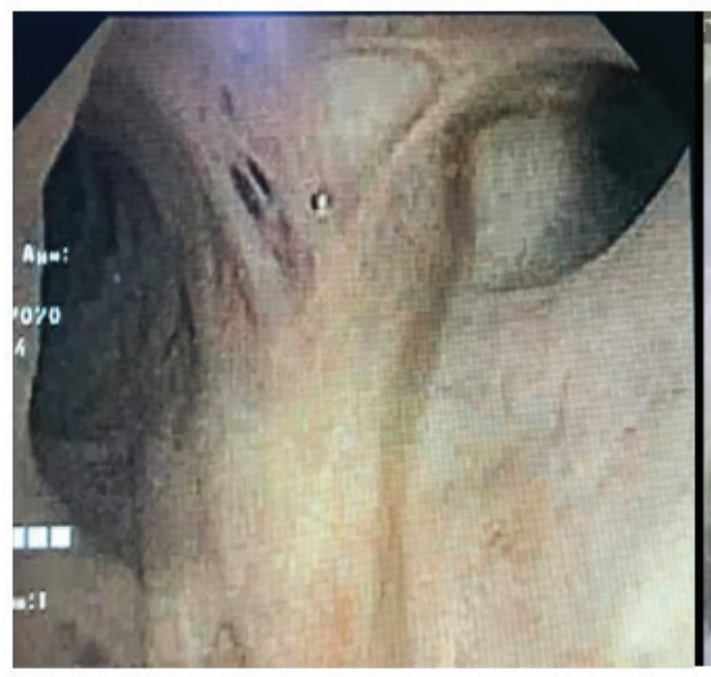

Fig 3.Bronchoscopy: Blood in RUL

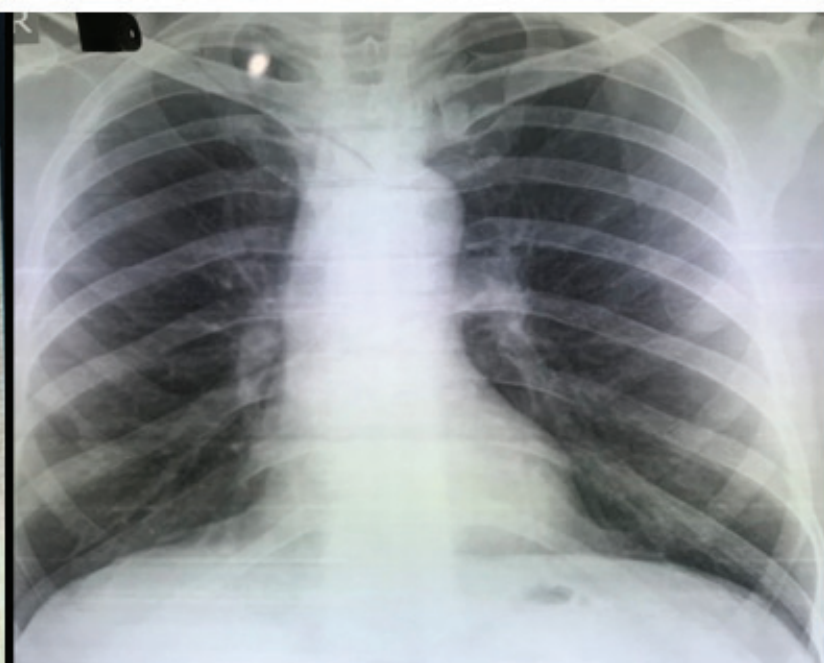

Fig 4: Resolved RUL consolidation

\section{Discussion}

Citrobacter species are straight, facultative anaerobic, Gram-negative bacilli and are typically motile by means of peritrichous flagellae.. This genus was proposed in 1932 by Werkman and Gillen.. In year 1993, Brenner et al. classified Citrobacter into 11 genomic species by DNA hybridization. The leading three species causing human infections are C. freundii, C. koseri, and C. Braakii ${ }^{7}$. Citrobacter species are commonly found in water, soil, food, and the intestinal tracts of animals and humans ${ }^{2}$. Two groups of patients are at risk of acquiring Citrobacter infections. The first one is neonates, who may develop sepsis and meningitis and brain abscess (usually less than 2 months of age). The second group is debilitated, aged ( $\geq 65$ years) or immunocompromised patients ${ }^{5}$. The infection may occur as sporadic cases or nosocomial outbreaks. Among the various sites of infection, the urinary tract is the most common, followed by the abdomen, skin/soft tissues (including surgical site infection), and pneumonia. A large surveillance study demonstrated that $0.8 \%$ of Gramnegative infection was caused by Citrobacter spp. ${ }^{3}$. In 
the hospital settings, Citrobacter spp. might account for 3-6\% of all Enterobacteriaceae causing nosocomial infection.

Mohanty etal from a tertiary care hospital in northern India, isolated Citrobactor from routine diagnostic testings from 205 patients from Jan till Dec 2004 .They observed that infection was nosocomially acquired in $94.6 \%$ patients. One hundred eighty one $(88.3 \%)$ patients had significant underlying illnesses. Culture yielded Citrobacter koseri in $185(90.2 \%)$ and Citrobacter freundii in $20(9.8 \%)$ patients. The distribution of isolates was as follows: urine $(46.2 \%)$, respiratory tract $(16.3 \%)$, blood (15.8\%), pus (12.1\%) and sterile body fluids $(9.3 \%)$. Drug resistance was observed to be more in C. koseri as compared to C. Freundii ${ }^{11}$

Ashwin Songara et al from India studied role of bronchoscopy in evaluation of patients with hemoptysis. They found Citrobactor freundi to grow in bronchial washings in $2.85 \%$ of patients ${ }^{6}$.

In a study of community acquired pneumonia in elderly $(>65$ yrs $)$ Anupam Pillai etal reported Citrobactor freundii as causative agent in 2 cases out of 65 study patients $^{8}$

Miguel Angel Ariza-Prota etal in year 2015 reported pneumonia and empyema in a72 year old Spanish male and claimed to be first reported case of community acquired pneumonia in a immunocompetent male caused by Citrobactor koseri 9 .

Kelly Pennington et al from Mayo clinic Rochester in year 2016 reported pneumonia attributable to Citrobactor koseri in 60year old man who on further investigation was found to have adenocarcinoma of lung ${ }^{10}$.

To the authors knowledge, this is the first reported case in the literature where a young immunocompetent male presents with community acquired pneumonia and haemoptysis caused by Citrobactor freundii.

Patients with Citrobacter infection can be identified and confirmed only by culture. Different species can be differentiated by biochemical tests ${ }^{2}$. The treatmentof Citrobacter infections follows the principles for treatment of other Enterobacteriaceae infections. Aminoglycosides, fluoroquinolones, carbapenems, and the fourth-generation cephems, such as cefepime and cefpirome, are the preferred therapeutic agents for $C$. freundii infections. Once a specific strain is isolated from a patient, therapeutic agents should be selected according to the in vitro susceptibility results of that strain.The, the site of infection also influences choice of agents ${ }^{12}$.

\section{Conclusion}

Citrobactor freundi causing community acquired pneumonia presenting as hemoptysis in a immunocompetent host is a rare infection. Culture and sensitivity of the sample from involved organ is the key to definitive diagnosis and successful treatment.

\section{Ethical Clearance: NA}

\section{Conflict of Interest: Nil}

\section{Source of Funding: Self}

\section{References}

1. Janda J. M., Abbott S. L., Cheung W. K. W., Hanson D. F. Biochemical identification of citrobacteria in the clinical laboratory. Journal of Clinical Microbiology. 1994;32(8):1850-1854

2. Arens S., Verbist L. Differentiation and susceptibility of Citrobacter isolates from patients in a university hospital. Clinical Microbiology and Infection. 1997;3(1):53-57

3. Schwartz D. A. Citrobacter infections. In: Connor D. H., Chandler F. W., Schwartz D. A., Manz H. J., Lack E. E., editors. Pathology of Infectious Diseases. Stanford, Connecticut: Appleton and Lange. 1997. pp. 513-516

4. Samonis G, Karageorgopoulos DE, Kofteridis DP, Matthaiou DK, Sidiropoulou V, Maraki S, Falagas ME. Citrobacter infections in a general hospital: characteristics and outcomes. Eur J Clin Microbiol Infect Dis 2009;28:61-68

5. Lipsky BA, Hook EW III, Smith AA, Plorde JJ. Citrobacter infections in humans: experience at the Seattle Veterans Administration Medical Center and a review of the literature. Rev Infect Dis 1980;2:746-760

6. Ashwin Songara, Rohit Mukherjee, Swapnil Shikha, Awadesh P. S. Solanki, Tanay Joshi, Deepika Patel.Role of fiberoptic bronchoscopy in 
evaluation of hemoptysis. Int J Res Med Sci. 2016

Jul;4(7):2576-2582

7. BrennerDJ, GrimontPAD, Steigerwalt AG, Fanning GR, Ageron E, Riddle CF. Classification of Citrobacteria by DNA hybridization. Int $J$ Syst Bacteriol 1993; 43: 645- 58

8. Anupa Pillai, Shwet Sabnis, Tushar Kanti Biswas. A study of Community Acquired Pneumonia in the Elderly. IAIM, 2019; 6(12): 1-9.

9 Miguel Angel Ariza-Prota, Ana PandoSandoval, Marta García-Clemente, Ramón Fernández, and Pere Casan. Community-Acquired Pneumonia and Empyema Caused by Citrobacter koseri in an Immunocompetent Patient.Case
Reports in Pulmonology Vol 2015.pp:1-6

10. Kelly Penington,Martin Van Zyl,Patricio Escalante. Citrobactor koseri pneumonia as initial presentation of underlying pulmonary adenocarcinoma.Clin Med Insights Case Rep 2016;9:87-89

11. Mohanty S., Singhal R., Sood S., Dhawan B., Kapil A., Das B. K. Citrobacter infections in a tertiary care hospital in Northern India. Journal of Infection. 2007;54(1):58-64.

12. Shih CC, Chen YC, Chang SC, Luh KT, Hsieh WC. Bacteremia due to Citrobacter species: significance of primary intraabdominal infection. Clin Infect Dis 1996;23:543-549. 